\title{
Preface
}

\section{A Contemporary Approach to Soft Tissue Tumors}

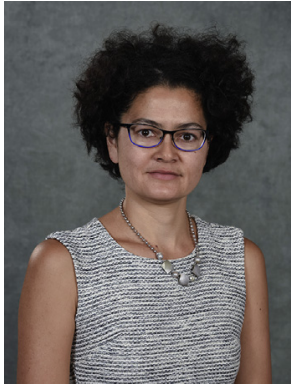

Elizabeth G. Demicco, MD, PhD Editor

Soft tissue sarcomas are a diverse and diagnostically challenging group of malignancies that may arise at any anatomic site. Classification of these tumors relies on morphology (e.g. spindle cell, round cell, epithelioid, or pleomorphic), line of differentiation (e.g. smooth muscle, vascular, adipocytic, skeletal muscle, nerve sheath, or unrelated to normal tissue lineages), and genomic features (specific gene-fusion, mutation driven, complex karyotype), and the differential diagnosis may vary depending on the tumor site, patient age, and sex. These classification systems overlap and interact in complex ways, such that tumors of myogenic differentiation, for instance, may be classified as mutation associated (a subset of spindle cell/sclerosing rhabdomyosarcoma), translocation driven (alveolar rhabdomyosarcoma, another subset of spindle cell/sclerosing rhabdomyosarcoma), or complex karyotype (pleomorphic rhabdomyosarcoma). Moreover, some sarcomas can behave differently based on site of origin (uterine vs soft tissue leiomyosarcoma), while multiple diverse tumors share EWSR1-related gene fusions as common driver event and may display overlapping morphologic features, while showing very different clinical behaviors (e.g., soft tissue myoepithelioma and extraskeletal myxoid chondrosarcoma).

This issue of Surgical Pathology Clinics breaks down these complex, interlocking topics into a series of articles focused on tissue lineage of differentiation, predominant morphology, organ of origin, or characteristic molecular event, in order to help readers contextualize these tumors within their histologic, anatomic, or molecular differential diagnosis. These reviews contain state-of-the-art updates on the molecular biology of sarcomas discovered over the past decade, including the identification of specific alterations, contemporary theories on how these alterations drive sarcomagenesis, diagnostic tests derived from these molecular alterations, and in some cases, how changing technologies enabled these discoveries. In addition, the current understanding of the role of prognostic and therapeutic biomarkers in sarcomas is explored, although many proposed biomarkers have yet to reach widespread clinical acceptance. Finally, the diagnosis of mesenchymal tumors in this age of increasingly smaller diagnostic biopsies is reviewed, with a practical focus on what is, and is not, achievable on limited tissue samples.

It is hoped that this issue will serve both as a diagnostic aid and an updated reference for the biology of soft tissue sarcomas.

Elizabeth G. Demicco, MD, PhD Department of Pathology and Laboratory Medicine

Mount Sinai Hospital 600 University Avenue

Toronto, ON, M5G 1X5, Canada

Department of Laboratory Medicine and Pathobiology

University of Toronto Toronto, ON M5S 1A1, Canada

E-mail address: elizabeth.demicco@sinaihealthsystem.ca 